\title{
Fainting matters: a case of an autoimmune polyglandular syndrome (APS) with an atypical involvement of the parathyroid gland
}

\author{
leva Ruža ${ }^{1,2,3}$, Sabīne Upmale ${ }^{3}$, Justīne Mālinga ${ }^{3}$, Inta Leitāne² \\ ${ }^{1}$ Department of Internal Medicine, Riga Eastern Clinical University Hospital, Latvia \\ ${ }^{2}$ Tornakalna Outpatient Clinic, Riga, Latvia \\ ${ }^{3}$ Riga Stradins University, Latvia
}

Introduction: The autoimmune polyglandular syndrome (APS) is characterized by the coexistence of at least two glandular

autoimmune mediated diseases.

We report a combination of an autoimmune thyroid disease, Addison's disease and an atypical involvement of the parathyroid gland.

\section{First visit:}

A 73-years old white woman was admitted to the clinic for evaluation due to recurrent syncopes during the previous 3 years that presented along with:

- vertigo

- cold sweats

- general fatigue

and was associated with prolonged standing.

- Loss of $15 \mathrm{~kg}$ weight within the past year - No skin changes observed

3 months ago she was diagnosed with:

- Chronic autoimmune thyroiditis and primary hypothyroidism

- Parathyroid gland adenoma (SPECT-CT)

- Orthostatic hypotension

\section{Investigations:}

- Head CT scan - basal ganglia calcinosis

- Head MRI, EEG, brachiocephalic vessel duplex ultrasonography, Holter monitoring revealed no substantial changes

- DXA scan - lumbar T-score -3,1 SD

- Antibody screen (ANA, thyroid peroxidase, transglutaminase) was negative.

- Low cortisol in 24h urine

- No data on diabetes mellitus or malignancy

\begin{tabular}{|l|l|}
\hline Aldosterone (40 - 310 pg/ml) & 3.7 \\
\hline Renin (4.4-46.1 mU/l) & 2.1 \\
\hline ACTH (7.2 - 63.3 pg/ml) & 44.5 \\
\hline Cortisol 8:00 (3.7-19.4 mkg/dl) & 3.7 \\
\hline TSH (0.35 - 4.94 mU/L) & 12 \\
\hline FT4 (0.71 - 1.85 ng/dl) & 0.95 \\
\hline Calcium (2.1 - 2.55 mmol/l) & 2.03 \\
\hline PTH (15 - 68 pg/ml) & 62.4 \\
\hline Creatinine (62 - 106 mkmol/L) & 95 \\
\hline
\end{tabular}

Insulin stress test insufficient rise

\begin{tabular}{|l|l|}
\hline $\begin{array}{l}\text { Plasma cortisol } \\
\text { at } 8: 00 \\
(3.7-19.4 \mathrm{mkg} / \mathrm{dl})\end{array}$ & 3.7 \\
\hline $\begin{array}{l}30 \mathrm{~min} \text { after } \\
\text { infusion }\end{array}$ & 4.1 \\
\hline $\begin{array}{l}60 \text { min after } \\
\text { infusion }\end{array}$ & 4.4 \\
\hline $\begin{array}{l}15 \text { min after } \\
\text { hypoglycemia }\end{array}$ & 10.0 \\
\hline $\begin{array}{l}60 \text { min after } \\
\text { hypoglycemia }\end{array}$ & 12.9 \\
\hline
\end{tabular}



Diagnosis:
- Primary adrenal insufficiency with glucocorticoid and
mineralocorticoid deficiency
- Chronic autoimmune thyroiditis with primary hypothyroidism
- Hypocalcaemia
- Calcinosis of basal ganglia (possible Fahr's syndrome)
- Parathyroid gland adenoma
- Secondary osteoporosis due to malabsorption
- Chronic kidney disease
Based on these findings, type 2 autoimmune polyglandular
syndrome was diagnosed.

\section{Conclusions:}

- PTH level was normal, but the coexistance of hypocalcaemia and parathyroid gland adenoma must be taken into consideration

- We would like to stress the importance of investigation for autoimmune glandular diseases and electrolyte level in case of unexplained syncope.

Contact e-mail: dr.ieva.ruza@gmail.com
Treatment:

$$
\begin{aligned}
& \text { - L-Thyroxine } \\
& \text { - Oral calcium } \\
& \text { - Alphacalcidol } \\
& \text { - Hydrocortisone } \\
& \text { - Fludrocortisone }
\end{aligned}
$$

A substantial improvement of symptoms was seen in a control visit after 3 months 